\title{
SUPERSYMMETRY CONTRIBUTION TO BOTTOM QUARK PRODUCTION AT HADRON COLLIDERS
}

\author{
EDMOND L. BERGER \\ High Energy Physics Division, Argonne National Laboratory, Argonne, IL 60439, USA \\ E-mail: berger@anl.gov
}

\begin{abstract}
A new contribution from pair-production of light gluinos, of mass 12 to $16 \mathrm{GeV}$, with two-body decays into bottom quarks and light bottom squarks, helps to obtain a bottom-quark production rate in better agreement with hadron collider data. The masses of the gluino and bottom squark are further restricted by the ratio of like-sign to opposite-sign leptons at hadron colliders. Constraints on this scenario from other data are examined and predictions are made.
\end{abstract}

\section{Motivation and Constraints}

The cross section for bottom-quark production at hadron collider energies exceeds the central value of predictions of next-to-leading order (NLO) perturbative quantum chromodynamics (QCD) by about a factor of twot. This longstanding discrepancy has resisted_fully satisfactory resolution within the standard model 26. The NLO contributions are large, and it is not excluded that further higher-order effects in production and/or fragmentation may resolve the discrepancy. However, the disagreement is surprising because the relatively large mass of the bottom quark sets a hard scattering scale at which fixedorder perturbative QCD computations of other processes are generally successful. The data invite the possibility of a contribution from "new physics".

In a recent paper $\mathrm{B}$, my collaborators and I explore an explanation within the context of the minimal supersymmetric standard model. We postulate the existence of a relatively light gluino $\tilde{g}$ (mass $\simeq 12$ to $16 \mathrm{GeV}$ ) that decays into a bottom quark $b$ and a light bottom squark $\tilde{b}$ (mass $\simeq 2$ to $5.5 \mathrm{GeV}$ ). The $\tilde{g}$ and the $\tilde{b}$ are the spin- $1 / 2$ and spin- 0 supersymmetric partners of the gluon $(g)$ and bottom quark. In our scenario the masses of all other supersymmetric particles are arbitrarily heavy, i.e., of order the electroweak scale or greater. While the gluino decays instantly with $100 \%$ branching fraction into the $b$ and $\tilde{b}$, the $\tilde{b}$ is either long-lived or decays via R-parity violation into a pair of hadronic jets. We obtain improved agreement with hadron collider rates of bottom-quark production. Several predictions are made that can be tested readily with forthcoming data.

Our assumptions are consistent with all constraints on the masses and couplings of supersymmetric parti-

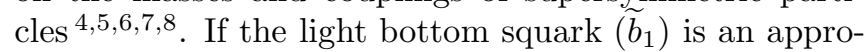
priate mixture of left-handed and right-handed bottom squarks, its tree-level coupling to the neutral gauge boson $Z$ can be small, feading to good agreement with the $Z$ peak observables 1 . The couplings $Z_{\tilde{b}_{1} \tilde{b}_{2}}$ and $Z_{\tilde{b}_{2} \tilde{b}_{2}}$, where $\widetilde{b}_{2}$ is the heavier bottom squark, survive but present no difficulty if $m_{\tilde{b}_{2}}>200 \mathrm{GeV}$. Bottom squarks make a small contribution to the inclusive cross section for $e^{+} e^{-} \rightarrow$ hadrons, in comparison to the contributions from quark production, and $\tilde{b} \tilde{b}$ resonances are likely to be impossible to extract from backgrounds 5 . The angular distribution of hadronic jets produced in $e^{+} e^{-}$annihilation can be examined in order to bound the contribution of scalar-quark production. Spin- $1 / 2$ quarks and spin-0 squarks emerge with different distributions, $\left(1 \pm \cos ^{2} \theta\right)$, respectively. We re-fit the angular distribution measured by the CELLO collaboration 6 , and find it is consistent with the production of a single pair of charge- $1 / 3$ squarks along with five flavors of quarkantiquark pairs. A new examination of the angular distribution with greater statistics would be valuable. The exclusion by the CLEO collaboration $\theta$ of a $\tilde{b}$ with mass 3.5 to $4.5 \mathrm{GeV}$ does not apply since that analysis focuses on the decays $\tilde{b} \rightarrow c l \tilde{\nu}$ and $\tilde{b} \rightarrow c l$. On the other hand, these data might be reinterpreted in terms of a bound on the R-parity violating lepton-number violating decay of $\tilde{b}$ into $\mathrm{cl}$. It would be interesting to study the hadronic decays $\tilde{b} \rightarrow c q$, with $q=d$ or $s$, and $\tilde{b} \rightarrow u s$ with the CLEO data. In summary, measurements at $e^{+} e^{-}$colliders do not significantly constrain $\tilde{b}$ masses. An analysis of 2 - and 4 -jet events by the ALEPH collaboration 1 disfavors $\tilde{g}$ 's with mass $m_{\tilde{g}}<6.3 \mathrm{GeV}$ but not $\tilde{g}$ 's in the mass rapge of interest to us. A renormalization group argument suggests that the existence of a light $\tilde{b}$ goes hand-in-hand with a comparatively light $\tilde{g}\left(m_{\tilde{g}} \sim 10 \mathrm{GeV}\right)$.

\section{Differential Cross Section}

Because the excess production rate is observed in all bottom-quark decay channels, an explanation in terms of new physics is guided towards hypothesized new particles that decay either like bottom quarks or directly to bottom quarks. The former is difficult to implement successfully $B$. In our scenario, light gluinos are produced in pairs via standard QCD subprocesses, dominantly $g+g \rightarrow \tilde{g}+\tilde{g}$ at Tevatron and Large Hadron Collider (LHC) energies. 
The $\tilde{g}$ has a strong color coupling to $b$ 's and $\tilde{b}$ 's and, as long as its mass satisfies $m_{\tilde{g}}>m_{b}+m_{\tilde{b}}$, the $\tilde{g}$ decays promptly to $b+\tilde{b}$. The magnitude of the $b$ cross section, the shape of the $b$ 's transverse momentum $p_{T b}$ distribution, and the CDF measurement 10 of $B^{0}-\bar{B}^{0}$ mixing are three features of the data that help to establish the preferred masses of the $\tilde{g}$ and $\tilde{b}$.

We include contributions from both $q+\bar{q} \rightarrow \tilde{g}+\tilde{g}$ and $g+g \rightarrow \tilde{g}+\tilde{g}$. The subprocess $g+b \rightarrow \tilde{g}+\tilde{b}$ contributes insignificantly. In Fig. 1 we show the integrated $p_{T b}$ distribution of the $b$ quarks that results from $\tilde{g} \rightarrow b+\tilde{b}$, for $m_{\tilde{g}}=14 \mathrm{GeV}$ and $m_{\tilde{b}}=3.5 \mathrm{GeV}$. The results are compared with the cross section obtained from next-to-leading order (NLO) perturbative QCD and CTEQ4M parton distribution functions (PDF's) 11, with $m_{b}=4.75 \mathrm{GeV}$, and a renormalization and factorization scale $\mu=\sqrt{m_{b}^{2}+p_{T b}^{2}}$. SUSY-QCD corrections to $b \bar{b}$ production are not included as they are not available and are generally expected to be somewhat smaller than the standard QCD corrections. A fully differential NLO calculation of $\tilde{g}$-pair production and decay does not exist either. Therefore, we compute the $\tilde{g}$-pair cross section from the leading order (LO) matrix element with NLO PDF's 凹, $\mu=\sqrt{m_{\tilde{g}}^{2}+p_{T_{\tilde{g}}}^{2}}$, a two-loop $\alpha_{s}$, and multiply by 1.9 , the ratio of inclusive NLO to LO cross sections 12 .

A relatively light gluino is necessary in order to obtain a bottom-quark cross section comparable in magnitude to the pure QCD component. Values of $m_{\tilde{g}} \simeq$ 12 to $16 \mathrm{GeV}$ are chosen because the resulting $\tilde{g}$ decays produce $p_{T b}$ spectra that are enhanced primarily in the neighborhood of $p_{T b}^{\min } \simeq m_{\tilde{g}}$ where the data show the most prominent enhancement above the QCD expectation. Larger values of $m_{\tilde{g}}$ yield too little cross section to be of interest, and smaller values produce more cross section than seems tolerated by the ratio of like-sign to opposite-sign leptons from $b$ decay, as discussed below. The choice of $m_{\tilde{b}}$ has an impact on the kinematics of the $b$. After selections on $p_{T b}^{\min }$, large values of $m_{\tilde{b}}$ reduce the cross section and, in addition, lead to shapes of the $p_{T b}$ distribution that agree less well with the data. The values of $m_{\tilde{b}}$ and $m_{\tilde{g}}$ are correlated; similar results to those shown in Fig. 1 can be obtained with $m_{\tilde{g}} \simeq 12 \mathrm{GeV}$, but $m_{\tilde{b}} \simeq m_{b}$.

After the contributions of the NLO QCD and SUSY components are added, the magnitude of the bottomquark cross section and the shape of the integrated $p_{T b}^{\min }$ distribution are described well. A theoretical uncertainty of roughly $\pm 30 \%$ may be assigned to the final solid curve, associated with variation of the $b$ mass, the renormalization/factorization scale, and the parton distributions. The SUSY process produces bottom quarks in a fourbody final state and thus their momentum correlations are different from those of QCD. Angular correlations

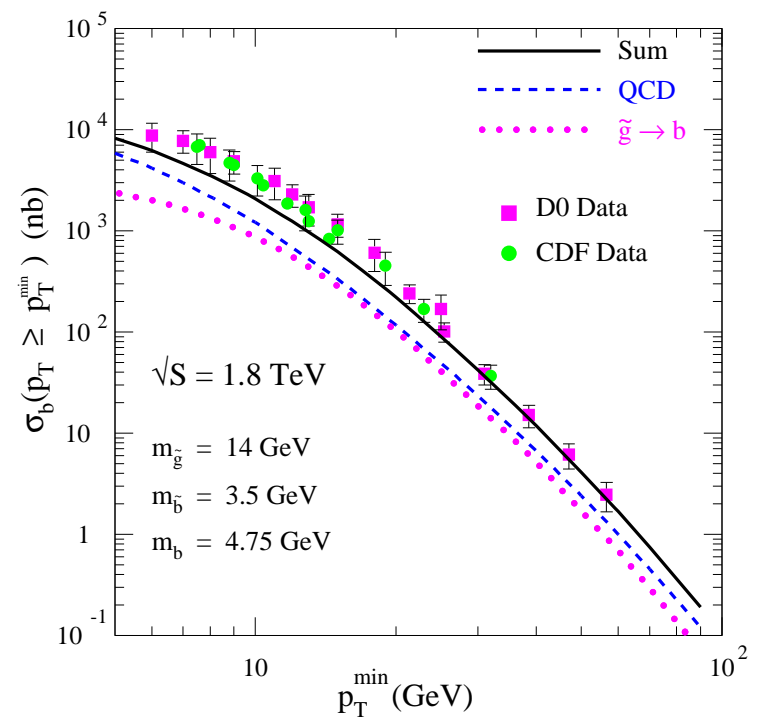

Figure 1: Bottom-quark cross section in $p \bar{p}$ collisions at $\sqrt{S}=1.8$ $\mathrm{TeV}$ for $p_{T b}>p_{T b}^{\min }$ with a gluino of mass $m_{\tilde{g}}=14 \mathrm{GeV}$ and a bottom squark of mass $m_{\tilde{b}}=3.5 \mathrm{GeV}$. The dashed curve is the central value of the NLO QCD prediction. The dotted curve shows the $p_{T}$ spectrum of the $b$ from the supersymmetry (SUSY) processes. The solid curve is the sum of the QCD and SUSY components. Data are from Ref. 1.

between mypns that arise from decays of $b$ 's have been measured 10.13 . Examining the angular correlations between $b$ 's in the SUSY case, we find they are nearly indistinguishable from those of QCD once experimental cuts are applied.

\section{Same-sign to Opposite-sign Leptons}

Since the $\tilde{g}$ is a Majorana particle, its decay yields both quarks and antiquarks. Gluino pair production and subsequent decay to $b$ 's will generate $b b$ and $\bar{b} \bar{b}$ pairs, as well as the $b \bar{b}$ final states that appear in QCD production. When a gluino is highly relativistic, its helicity is nearly the same as its chirality. Therefore, selection of $\tilde{g}$ 's whose transverse momentum is greater than their mass will reduce the number of like-sign $b$ 's. In the intermediate $p_{T}$ region, however, the like-sign suppression is reduced. The cuts chosen in current hadron collider experiments for measurement of the ratio of like-sign to opposite-sign muons result in primarily unpolarized $\tilde{g}$ 's, and, independent of the $\tilde{b}$ mixing angle, an equal number of like-sign and opposite-sign $b$ 's is expected at production. Our SUSY mechanism leads therefore to an increase of like-sign leptons in the final state after semi-leptonic decays of the $b$ and $\bar{b}$ quarks. This increase could be confused with an enhanced rate of $B^{0}-\bar{B}^{0}$ mixing. 
Time-integrated mixing analyses of lepton pairs observed at hadron colliders are interpreted in terms of the quantity $\bar{\chi}=f_{d} \chi_{d}+f_{s} \chi_{s}$, where $f_{d}$ and $f_{s}$ are the fractions of $B_{d}^{0}$ and $B_{s}^{0}$ hadrons in the sample of semi-leptonic $B$ decays, and $\chi_{f}$ is the time-integrated mixing probability for $B_{f}^{0}$. Conventional $b \bar{b}$ pair production determines the quantity $L S_{c}=2 \bar{\chi}(1-\bar{\chi})$, the fraction of $b \bar{b}$ pairs that decay into like-sign leptons. Our SUSY mechanism leads to a new expression

$$
L S=\frac{1}{2} \frac{\sigma_{\tilde{g} \tilde{g}}}{\sigma_{\tilde{g} \tilde{g}}+\sigma_{\mathrm{qcd}}}+L S_{c} \frac{\sigma_{\mathrm{qcd}}}{\sigma_{\tilde{g} \tilde{g}}+\sigma_{\mathrm{qcd}}}=2 \bar{\chi}_{\mathrm{eff}}\left(1-\bar{\chi}_{\mathrm{eff}}\right) .
$$

The factor $1 / 2$ arises because $N(b b+\bar{b} \bar{b}) \simeq N(b \bar{b})$ in the new mechanism. Introducing $G=\sigma_{\tilde{g} \tilde{g}} / \sigma_{\mathrm{qcd}}$, the ratio of SUSY and QCD bottom-quark cross sections after cuts, and solving for the effective mixing parameter, we obtain:

$$
\bar{\chi}_{\mathrm{eff}}=\frac{\bar{\chi}}{\sqrt{1+G}}+\frac{1}{2}\left[1-\frac{1}{\sqrt{1+G}}\right] .
$$

We interpret the CDF collaboration's measurement as a determination of $\bar{\chi}_{\mathrm{eff}}=0.131 \pm 0.02 \pm 0.016 \notin 0$ and note that it is marginally larger than the world average value $\bar{\chi}=0.118 \pm 0.0054$.

To estimate our theoretical $\bar{\chi}_{\text {eff }}$, we assume that $\bar{\chi}=0.118 \pm 0.00544$ represents the contribution from only the QCD $b \bar{b}$ component. We determine the ratio $G$ in the region of phase space where the measurement is made 0 , with both final b's having $p_{T b} \geq 6.5 \mathrm{GeV}$ and rapidity $\left|y_{b}\right| \leq 1$. For gluino masses of $m_{\tilde{g}}=14$ and $16 \mathrm{GeV}$, we obtain $G=0.37$ and 0.28 , respectively, with $m_{\tilde{b}}=3.5$ $\mathrm{GeV}$. We compute $\bar{\chi}_{\text {eff }}=0.17$ for $m_{\tilde{g}}=14 \mathrm{GeV}$, and $\bar{\chi}_{\text {eff }}=0.16$ with $m_{\tilde{g}}=16 \mathrm{GeV}$. To estimate the uncertainty on $G$, we vary the renormalization/factorization scale at which the cross sections are evaluated between $\mu=m_{x} / 2$ and $\mu=2 m_{x}$. Uncertainties of $\pm 50 \%$ are obtained and lead to uncertainties in $\bar{\chi}_{\text {eff }}$ of $\delta \bar{\chi}_{\text {eff }} \simeq \pm 0.02$. Additional theoretical uncertainties arise because there is no fully differential NLO calculation of gluino production and subsequent decay to $b$ 's.

Comparing our expectations with the CDF value, we conclude that values of $m_{\tilde{g}}>12 \mathrm{GeV}$ lead to a calculated $\bar{\chi}_{\text {eff }}$ that is consistent with the data within experimental and theoretical uncertainties. With $\sigma_{\tilde{g} \tilde{g}} / \sigma_{\mathrm{qcd}} \sim 1 / 3$, we can satisfy the magnitude and $p_{T}$ dependence of the $b$ production cross section and the mixing data.

\section{Implications and Remarks}

\subsection{Hadron Reactions}

Among the predictions of this SUSY scenario, the most clearcut is pair production of like-sign charged $B$ mesons at hadron colliders, $B^{+} B^{+}$and $B^{-} B^{-}$. To verify the underlying premise of this work, that the cross section exceeds expectations of conventional perturbative QCD, a new measurement of the absolute rate for $b$ production in run II of the Tevatron is important. A very precise measurement of $\bar{\chi}$ in run II is obviously desirable. Since the fraction of $b$ 's from gluinos changes with $p_{T b}$, we also expect a change of $\bar{\chi}$ with the cut on $p_{T b}$. The $b$ jet from $\tilde{g}$ decay into $\vec{b} \vec{b}$ will contain the $\widetilde{b}$, implying unusual material associated with the $\widetilde{b}$ in some fraction of the $b \bar{b}$ data sample. The existence of light $\widetilde{b}$ 's means that they will be pair-produced in partonic processes, leading to a slight increase $(\sim 1 \%)$ in the hadronic dijet rate. Our approach increases the $b$ production rate at HERA and in $\gamma \gamma$ collisions at LEP by a small amount, not enough perhaps if early-oxperimental indications in these cases are confirmed 15.16, but a full NLO study should be undertaken and better parton densities of photons are needed.

\subsection{Running of $\alpha_{s}$}

In the standard model, a global fit to all observables provides an indirect measurement of the strong coupling strength $\alpha_{s}$ at the scale of the $Z$ boson mass $M_{Z}$. The value $\alpha_{s}\left(M_{Z}\right) \simeq 0.119 \pm 0.006$ describes most observables properly 17 . A light $\tilde{g}$ with mass about $15 \mathrm{GeV}$ and a light $\tilde{b}$ slow the running of $\alpha_{s}$ and modify $\alpha_{s}\left(M_{Z}\right)$, determined by extrapolation from experiments performed at energies lower than $m_{\tilde{g}}$. The result is a shift of 0.007 in $\alpha_{s}$ derived from these experiments, to $\alpha_{s}\left(M_{Z}\right) \simeq 0.125$, at the upper edge of the uncertainty band. Greater precision on the determination of $\alpha_{s}\left(M_{Z}\right)$ from evolution would be valuable. Slower running of $\alpha_{s}(Q)$ also means a slower evolution of parton densities at small $x$, an effect that might be seen in HERA data for $Q>m_{\tilde{g}}$. Presence of a scalar $\tilde{b}$ in the proton breaks the Callan-Gross relation and yields a non-zero leading-twist longitudinal structure function $F_{L}(x, Q)$ at leading-order.

\section{$4.3 \widetilde{b}$-onia}

Possible bound states of bottom squark pairs could be seen as $J^{P}=0^{+}, 1^{-}, 2^{+}, \ldots$ mesonic resonances in $\gamma \gamma$ reactions and in $p \bar{p}$ formation, with masses in the 4 to $10 \mathrm{GeV}$ range. They could show up as narrow states in the $\mu^{+} \mu^{-}$invariant mass spectra at hadron colliders, between the $J / \Psi$ and $\Upsilon$. At an $e^{+} e^{-}$collider, the intermediate photon requires production of a $J^{P C}=1^{--}$ state. Bound states of low mass squarks with charge $2 / 3$ were studied with a potential modelE. The small leptonic widths were found to preclude bounds for $m_{q}>3 \mathrm{GeV}$. For bottom squarks with charge $-1 / 3$, the situation is more difficult. 


\section{$4.4 \widetilde{b}$ lifetime and observability}

Strict R-parity conservation in the MSSM forbids $\widetilde{b}$ decay unless there is a lighter supersymmetric particle. R-parity-violating and lepton-number-violating decay of the $\widetilde{b}$ into at least one lepton is disfavored by the CLEO data 7 and would imply the presence of an extra lepton, albeit soft, in some fraction of $b$ jets observed at hadron colliders. The baryon-number-violating Rparity-violating $\left(R_{p}\right)$ term in the MSSM superpotential is $\mathcal{W}_{R_{p}}=\lambda_{i j k}^{\prime \prime} U_{i}^{c} D_{j}^{c} D_{k}^{c} ; U_{i}^{c}$ and $D_{i}^{c}$ are right-handedquark singlet chiral superfields; and $i, j, k$ are generation indices. The limits on individual $R_{p}$ and baryonnumber violating couplings $\lambda^{\prime \prime}$ are relatively weak for third-generation squarks 18 , $\lambda_{i j k}^{\prime \prime}<0.5$ to 1 .

The possible $R_{p}$ decay channels for the $\tilde{b}$ are 123 : $\overline{\widetilde{b}} \rightarrow u+s ; 213: \overline{\widetilde{b}} \rightarrow c+d$; and $223: \overline{\widetilde{b}} \rightarrow c+s$. The hadronic width is 19

$$
\Gamma(\widetilde{b} \rightarrow \text { jet }+ \text { jet })=\frac{\mathrm{m}_{\widetilde{\mathrm{b}}}}{2 \pi} \sin ^{2} \theta_{\widetilde{\mathrm{b}}} \sum_{\mathrm{j}<\mathrm{k}}\left|\lambda_{\mathrm{ij} 3}^{\prime \prime}\right|^{2} .
$$

If $m_{\tilde{b}}=3.5 \mathrm{GeV}, \Gamma(\widetilde{b} \rightarrow i j)=0.08\left|\lambda_{i j 3}^{\prime \prime}\right|^{2} \mathrm{GeV}$. Unless all $\lambda_{i j 3}^{\prime \prime}$ are extremely small, the $\tilde{b}$ will decay quickly and leave soft jets in the cone around the $b$. $b$-jets with an extra $c$ are possibly disfavored by CDF, but a detailed simulation is needed.

If the $\widetilde{b}$ is relatively stable, the $\widetilde{b}$ could pick up a light $\bar{u}$ or $\bar{d}$ and become a $\widetilde{B}^{-}$or $\widetilde{B}^{0}$ "mesino" with $J=1 / 2$, the superpartner of the $B$ meson. The mass of the mesino would fall roughly in the range 3 to $7 \mathrm{GeV}$ for the interval of $\widetilde{b}$ masses we consider. The charged mesino could fake a heavy muon if its hadronic cross section is small and if it survives passage through the hadron calorimeter and exits the muon chambers. Extra muon-like tracks would then appear in a fraction of the $b \bar{b}$ event sample, but tracks that left some activity in the hadron calorimeter. The mesino has baryon number zero but acts like a heavy $\bar{p}$ - perhaps detectable with a time-of-flight apparatus. A long-lived $\widetilde{b}$ is not excluded by conventional_searches at hadron and lepton colliders, but an analysis 20 similar to that for $\tilde{g}$ 's should be done to verify that there are no additional constraints on the allowed range of $\tilde{b}$ masses and lifetimes.

\section{Acknowledgments}

I am indebted to Brian Harris, David E. Kaplan, Zack Sullivan, Tim Tait, and Carlos Wagner for their collaboration and excellent suggestions. I have benefitted from valuable discussions with Barry Wicklund, Tom LeCompte, and Harry Lipkin. I congratulate Tony Sanda and his colleagues for organizing an excellent Fourth International Workshop on B Physics and CP Violation in an exceptional location. This research was supported by the U.S. Department of Energy under Contract W-31109-ENG-38.

\section{References}

1. CDF Collaboration, F. Abe et al., Phys. Rev. Lett. 71, 500 (1993); ibid 79, 572 (1997); ibid 79, 572 (1997); ibid 75, 1451 (1995); D0 Collaboration, B. Abbott et al., Phys. Lett. B487, 264 (2000) and Phys. Rev. Lett. 85, 5068 (2000).

2. P. Nason et al., in Proceedings of the 1999 CERN Workshop on Standard Model Physics (and more) at the $\mathrm{LHC}$, edited by G. Altarelli and M. L. Mangano, CERN Yellow Report No. CERN 2000-004, p. 231.

3. E. L. Berger, B. W. Harris, D. E. Kaplan, Z. Sullivan, T. Tait, and C. E. M. Wagner, hepph/0012001, to be published in Phys. Rev. Lett..

4. M. Carena, S. Heinemeyer, C. E. M. Wagner, and G. Weiglein, hep-ph/0008023, to be published in Phys. Rev. Lett..

5. C. R. Nappi, Phys. Rev. D 25, 84 (1982); S. Pacetti and Y. Srivastava, hep-ph/0007318.

6. CELLO Collaboration, H.-J. Behrend et al., Phys. Lett. B183, 400 (1987).

7. CLEO Collaboration, V. Savinov et al., Phys. Rev. D 63, 051101 (2001).

8. ALEPH Collaboration, R. Barate et al., Z. Phys. C76, 1 (1997).

9. A. Dedes and H. K. Dreiner, hep-ph/0009001.

10. CDF Collaboration, F. Abe et al., Phys. Rev. D 55, 2546 (1997).

11. CTEQ Collaboration, H. L. Lai et al., Phys. Rev. D 55, 1280 (1997).

12. W. Beenakker, R. Hopker, M. Spira, P. M. Zerwas, Nucl. Phys. B492, 51 (1997); W. Beenakker, R. Hopker, and M. Spira, hep-ph/9611232.

13. D0 Collaboration, B. Abbott et al., Phys. Lett. B487, 264 (2000) and references therein.

14. Particle Data Group, Eur. Phys. Jour. C15 1, (2000).

15. H1 Collaboration, C. Adloff et al., Phys. Lett. B467, 156 (1999); ZEUS Collaboration, J. Breitweg et al., hep-ex/0011081.

16. L3 Collaboration, M. Acciarri et al., hepex/0011070; OPAL Collaboration, A. Csilling et al., Photon 2000, Lancaster University, UK, 2000.

17. S. Bethke, J. Phys. G26, R27 (2000).

18. B. C. Allanach, A. Dedes, and H. K. Dreiner, Phys. Rev. D 60, 075014 (1999).

19. E. L. Berger, B. W. Harris, and Z. Sullivan, Phys. Rev. Lett. 83, 4472 (1999) and hep-ph/0012184, to be published in Phys. Rev. D.

20. H. Baer, K. Cheung, and J. F. Gunion, Phys. Rev. D 59, 075002 (1999). 